\title{
A Magnetic Type Integral Operator which is Stable till Extremely Low Frequencies
}

\author{
Francesco P. Andriulli ${ }^{1}$, Ignace Bogaert ${ }^{2}$, Kristof Cools $^{3}$, and Eric Michielssen ${ }^{4}$ \\ ${ }^{1}$ École Nationale Supérieure des Télécommunications de Bretagne, e-mail: \\ francesco.andriulli@telecom-bretagne.eu \\ ${ }^{2}$ Ghent University, e-mail: ignace.bogaert@ugent.be \\ ${ }^{3}$ University of Nottingham, e-mail: kristof.cools@nottingham.ac.uk \\ ${ }^{4}$ University of Michigan at Ann Arbor, e-mail: emichiel@umich.edu
}

\begin{abstract}
We introduce a new magnetic field integral equation that does not suffer from low-frequency numerical cancelations even for extremely low frequencies $\left(10^{-40} \mathrm{~Hz}\right)$. The new equation is obtained by symmetrizing the standard MFIE with its dual equation and by using appropriately chosen quasi-Helmholtz projectors. When compared to mixed discretized MFIEs, the new equation maintains the favorable properties of mixed discretizations even without using high precision integration rules. Numerical results confirm the theoretical developments and show the effectiveness of the new scheme.
\end{abstract}

\section{Introduction}

All known integral equation techniques for simulating scattering and radiation from arbitrarily shaped, perfect electrically conducting objects suffer from one or more of the following shortcomings: (i) they give rise to ill-conditioned systems when the frequency is low (ii) and/or when the discretization density is high; (iii) their applicability is limited to the quasi-static regime; (iv) they require a search for global topological loops; (v) they suffer from numerical cancelations in the solution when the frequency is very low. A recent paper [1] presented a new integral operator of the electric type that does not suffer from any of the above drawbacks.

This contribution complements the recently developed electric type operator immune from (i)-(v) by developing a new magnetic type operator. The new formulation is obtained starting from a Helmholtz decomposition of two discretizations of the electric field integral operator and from a suitably symmetrized mixed discretization of the magnetic field integral operator obtained by using RWGs and dual bases functions, respectively. The discretization of the new formulation is analyzed in detailed and it is shown that the new equation maintains the favorable properties of the mixed discretization even without using high precision integration rules. Numerical results confirm the theoretical developments and show the effectiveness of the scheme. Some preliminary applications of this new equation have been presented in [2].

\section{Background and Formulation}

Consider the surface $\Gamma$ of a closed PEC object residing in a space of permittivity $\epsilon$ and permeability $\mu$. Denote with $\hat{\boldsymbol{n}}_{\boldsymbol{r}}$ its outward pointing unit normal at $\boldsymbol{r}$. Denote with $\Omega^{+}$and $\Omega^{-}$the exterior and interior regions of $\Gamma$ respectively. An incident electromagnetic field $\left(\boldsymbol{E}^{i}(\boldsymbol{r}), \boldsymbol{H}^{i}(\boldsymbol{r})\right)$ is impinging on $\Gamma$ inducing a surface current density $\boldsymbol{J}(\boldsymbol{r})$. The current $\boldsymbol{J}(\boldsymbol{r})$ can be retrieved by solving the Magnetic Field Integral Equation (MFIE)

$$
\left(\frac{\mathcal{I}}{2}+\mathcal{K}\right)(\boldsymbol{J})=\eta\left(\hat{\boldsymbol{n}}_{\boldsymbol{r}} \times \boldsymbol{H}^{i}\right)
$$


where

$$
\mathcal{K}(\boldsymbol{J})=-\hat{\boldsymbol{n}}_{\boldsymbol{r}} \times \int_{\Gamma} \nabla \frac{e^{i k\left|\boldsymbol{r}-\boldsymbol{r}^{\prime}\right|}}{4 \pi\left|\boldsymbol{r}-\boldsymbol{r}^{\prime}\right|} \times \boldsymbol{J}\left(\boldsymbol{r}^{\prime}\right) \mathrm{d} \boldsymbol{r}^{\prime}
$$

where $k=2 \pi / \lambda=\omega \sqrt{\epsilon \mu}$ and $\eta=\sqrt{\mu / \epsilon}$. Note that with these definitions the current $\boldsymbol{J}(\boldsymbol{r})$ represents the jump over $\Gamma$ of the total magnetic field multiplied by the medium characteristic impedance.

The MFIE can be solved by using boundary elements, $\Gamma$ is approximated by a mesh of planar triangles with average edge length $h$, and $\boldsymbol{J}(\boldsymbol{r})$ is approximated as

$$
\boldsymbol{J}(\boldsymbol{r}) \approx \sum_{n=1}^{N} I_{n} \boldsymbol{f}_{n}(\boldsymbol{r})
$$

where $\boldsymbol{f}_{n}(\boldsymbol{r}), n=1, \ldots, N$ are Rao-Wilton-Glisson (RWG) div-conforming basis functions defined on the mesh's $N$ internal edges. To simplify the notation in what follows the RWGs are taken without the edge length normalization. To solve the MFIE (3) is substituted into (1) and the resulting equation is tested with the functions $\boldsymbol{f}_{n}$ yielding the $N \times N$ discretized MFIE system

$$
\left(\frac{\overline{\overline{\mathbf{G}}}}{2}+\overline{\overline{\mathbf{K}}}\right) \overline{\mathbf{I}}=\overline{\mathbf{V}}_{H}
$$

where $\overline{\overline{\mathbf{K}}}=\left\langle\boldsymbol{f}_{i}, \mathcal{K}\left(\boldsymbol{f}_{j}\right)\right\rangle,\left(\overline{\mathbf{V}}_{H}\right)_{i}=-\left\langle\boldsymbol{f}_{i}, \eta\left(\hat{\boldsymbol{n}}_{\boldsymbol{r}} \times \boldsymbol{H}^{i}\right)\right\rangle$, and

$$
(\overline{\overline{\mathbf{G}}})_{i, j}=\left\langle\boldsymbol{f}_{i}, \mathcal{I}\left(\boldsymbol{f}_{j}\right)\right\rangle=\left\langle\boldsymbol{f}_{i}, \boldsymbol{f}_{j}\right\rangle
$$

is the Gram matrix of the RWG basis. The MFIE is the sum of the identity and of an operator $(\mathcal{K})$ which is compact when $\Gamma$ is smooth. Operators that are the sum of the identity and of a compact operator are called second kind operators and matrices arising from their discretization are well-conditioned.

\section{A new magnetic operator and its discretization}

It has been shown that the standard discretization (4) of the MFIE leads to incorrect results at low frequencies [3]. The standard discretization of the MFIE uses the div-conforming RWGs both as source and testing functions. On the other hand, the mapping properties of the MFIE operator suggests that the MFIE testing functions should be curl-conforming basis functions. For this reason the mixed discretized MFIE has been proposed in [4] and further analyzed in [5] and [6]. In the mixed discretized MFIE, the testing functions are the Buffa-Christiansen (BC) or the Chen-Wilton (CW) basis functions defined on the barycentric refinement. In [6], in particular, it has been shown that that the mixed MFIE does not suffer from the low-frequencies inaccuracies observed in [3] for the standard MFIE. The mixed MFIE reads

$$
\left(\frac{\overline{\overline{\mathbf{G}}}_{m i x}^{T}}{2}+\overline{\overline{\mathbf{K}}}_{m i x}^{k}\right) \overline{\mathbf{I}}=\overline{\mathbf{V}}_{H}
$$

where $\left(\overline{\overline{\mathbf{K}}}_{\text {mix }}^{k}\right)_{i, j}=\left\langle\hat{\boldsymbol{n}}_{\boldsymbol{r}} \times \boldsymbol{f}_{i}^{B C}, \mathcal{K}_{k}\left(\boldsymbol{f}_{j}\right)\right\rangle$ is the mixed discretized MFIE operator, $\left(\overline{\overline{\mathbf{G}}}_{\text {mix }}\right)_{i, j}=$ $\left\langle\hat{\boldsymbol{n}}_{\boldsymbol{r}} \times \boldsymbol{f}_{i}, \boldsymbol{f}_{j}^{B C}\right\rangle$ is the mixed Gram matrix, $\left(\overline{\mathbf{V}}_{H}\right)_{i}=-\left\langle\hat{\boldsymbol{n}}_{\boldsymbol{r}} \times \boldsymbol{f}_{i}^{B C}, \eta\left(\hat{\boldsymbol{n}}_{\boldsymbol{r}} \times \boldsymbol{H}^{i}\right)\right\rangle$ is the magnetic 
right hand sides, and $(\overline{\mathbf{I}})_{j}=I_{j}$ is the unknown current vector such that $\boldsymbol{J}(\boldsymbol{r}) \approx \sum_{n=1}^{N} I_{n} \boldsymbol{f}_{n}(\boldsymbol{r})$. In $[5]$ it has been shown that, although the operator in (6) is stable at low frequencies when the matrices are computed in infinite precision, this would not be the case with finite precision computations. As a consequence, very precise integration rules would be required to stabilize the equation at very low frequencies. This problem will be solved in this contribution by a suitable discretization of a symmetrized magnetic operator.

The new symmetrized magnetic equation we propose reads:

$$
\eta^{2}\left(\frac{\mathcal{I}}{2}-\mathcal{K}_{i k}\right)\left(\frac{\mathcal{I}}{2}+\mathcal{K}_{k}\right)(k)(\boldsymbol{J})=\left(\frac{\mathcal{I}}{2}-\mathcal{K}_{i k}\right)\left(\hat{\boldsymbol{n}}_{\boldsymbol{r}} \times \boldsymbol{H}\right)
$$

The discretization we propose for it is the following:

$$
\eta^{2} \mathbb{M}\left(\frac{\overline{\mathbf{G}}_{m i x}^{T}}{2}-\overline{\overline{\mathbf{K}}}_{m i x}^{i k}\right)\left(\overline{\overline{\mathbf{G}}}_{m i x}^{T}\right)^{-1}\left(\frac{\overline{\overline{\mathbf{G}}}_{m i x}^{T}}{2}+\overline{\overline{\mathbf{K}}}_{m i x}^{k}\right) \overline{\overline{\mathbf{M}}} \overline{\overline{\mathbf{I}}}=\eta^{2} \mathbb{M}\left(\frac{\overline{\overline{\mathbf{G}}}_{m i x}^{T}}{2}-\overline{\overline{\mathbf{K}}}_{m i x}^{i k}\right)\left(\overline{\overline{\mathbf{G}}}_{m i x}^{T}\right)^{-1} \overline{\mathbf{V}}_{H}
$$

where $\alpha$ is a constant, $\overline{\overline{\mathbf{M}}}=\overline{\overline{\mathbf{P}}}^{\Lambda H} \frac{1}{\alpha}+i \overline{\overline{\mathbf{P}}}^{\Sigma} \alpha, \overline{\overline{\mathrm{M}}}=\overline{\overline{\mathrm{P}}}^{\Sigma H} \frac{1}{\alpha}+i \overline{\overline{\mathbb{P}}}^{\Lambda} \alpha$, and where the definition of the projectors $\overline{\bar{P}}_{S}$ and $\overline{\overline{\mathbf{P}}}_{\mathrm{S}}$ is omitted here for space limitations, but it can be found in [1]. We cannot profusely discuss on the reasons of low-frequency numerical stability of (8), but they can be deduced from the table below: where, after some manipulation, it can be seen that, after choosing



$\alpha=\sqrt{k}$, all the relevant blocks shows a frequency independent scaling and that the static harmonic null-space is numerically exact.

\section{Numerical Results}

The numerical tests involve a sphere of unit radius that is excited by a plane wave. The fact that the new equation is immune from the very low frequency current cancelation is confirmed by Fig. 1(a) which show the far field calculated using (8) at $10^{-40} \mathrm{~Hz}$. The advantage of the new equation with respect to a mixed discretization of the MFIE is shown in figure 1(b) where the new equation, in 


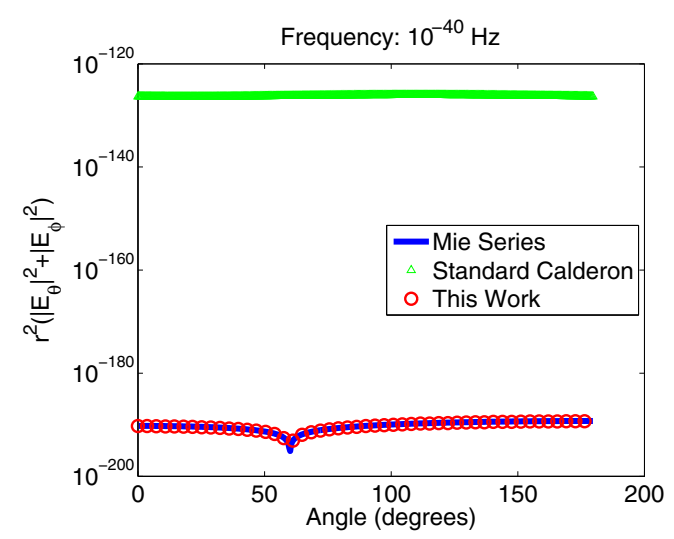

(a) Sphere: far field calculated when the frequency equals $10^{-40} \mathrm{~Hz}$.

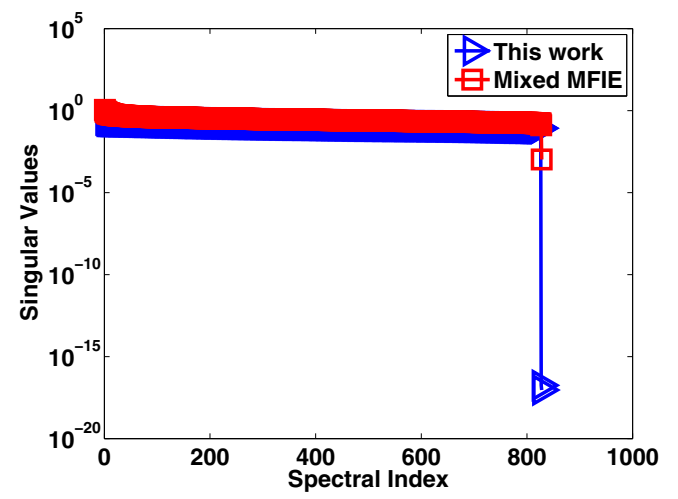

(b) Sphere: condition number as a function of frequency.

Figure 1: Numerical results

the case of a torus, show a machine precision null-space. On the contrary, the mixed discretized MFIE only shows a null-space level comparable to the integration precision.

\section{Acknowledgments}

This work has been partially funded by the ANR (Agence Nationale de la Recherche) under the project FASTEEG-ANR-12-JS09-0010. One of the authors also thank GENCI (Grand Equipement National de Calcul Intensif) for providing the necessary computing facilities for this work.

\section{References}

[1] F. Andriulli, K. Cools, I. Bogaert, and E. Michielssen, "On a well-conditioned electric field integral operator for multiply connected geometries," Antennas and Propagation, IEEE Transactions on, vol. 61, no. 4, pp. 2077-2087, April 2013.

[2] F. Andriulli, I. Bogaert, K. Cools, and E. Michielssen, "A well-conditioned combined field integral equation based on quasi-helmholtz projectors," in Electromagnetics in Advanced Applications (ICEAA), 2013 International Conference on, Sept 2013, pp. 644-647.

[3] Y. Zhang, T. J. Cui, W. C. Chew, and J.-S. Zhao, "Magnetic field integral equation at very low frequencies," Antennas and Propagation, IEEE Transactions on, vol. 51, no. 8, pp. 1864 1871, aug. 2003.

[4] K. Cools, F. Andriulli, F. Olyslager, and E. Michielssen, "Improving the mfie's accuracy by using a mixed discretization," in Antennas and Propagation Society International Symposium, 2009. APSURSI '09. IEEE, june 2009, pp. 1 -4.

[5] I. Bogaert, K. Cools, F. Andriulli, J. Peeters, and D. De Zutter, "Low frequency stability of the mixed discretization of the mfie," in Antennas and Propagation (EUCAP), Proceedings of the 5th European Conference on, april 2011, pp. $2463-2465$.

[6] I. Bogaert, K. Cools, F. Andriulli, and D. De Zutter, "Low frequency scaling of the mixed mfie for scatterers with a non-simply connected surface," in Electromagnetics in Advanced Applications (ICEAA), 2011 International Conference on, sept. 2011, pp. 951 -954. 